\title{
Erratum to: Comparison of corneal sensitivity between FS-LASIK and femtosecond lenticule extraction (ReLEx flex) or small-incision lenticule extraction (ReLEx smile) for myopic eyes
}

\author{
Shengsheng Wei • Yan Wang
}

Published online: 18 May 2013

(C) Springer-Verlag Berlin Heidelberg 2013

\section{Erratum to: Graefes Arch Clin Exp Ophthalmol DOI 10.1007/s00417-013-2272-0}

\section{Reason:}

Some figures and legends are mismatched - namely:

The figure printed as Fig 2 should read as Fig 4.

The figure printed as Fig 3 should read as Fig 5 .

The figure printed as Fig 4 should read as Fig 2.

The figure printed as Fig 5 should read as Fig 3.

The online version of the original article can be found at http:/ dx.doi.org/10.1007/s00417-013-2272-0.

S. Wei $\cdot$ Y. Wang $(\bowtie)$

Tianjin Eye Hospital \& Eye Institute, Tianjin Key Lab of Ophthalmology and Visual Science, Tianjin Medical University,

No 4. Gansu Rd, Heping District,

Tianjin 300020, China

e-mail: wangyan7143@vip.sina.com 


\section{Corrected version:}

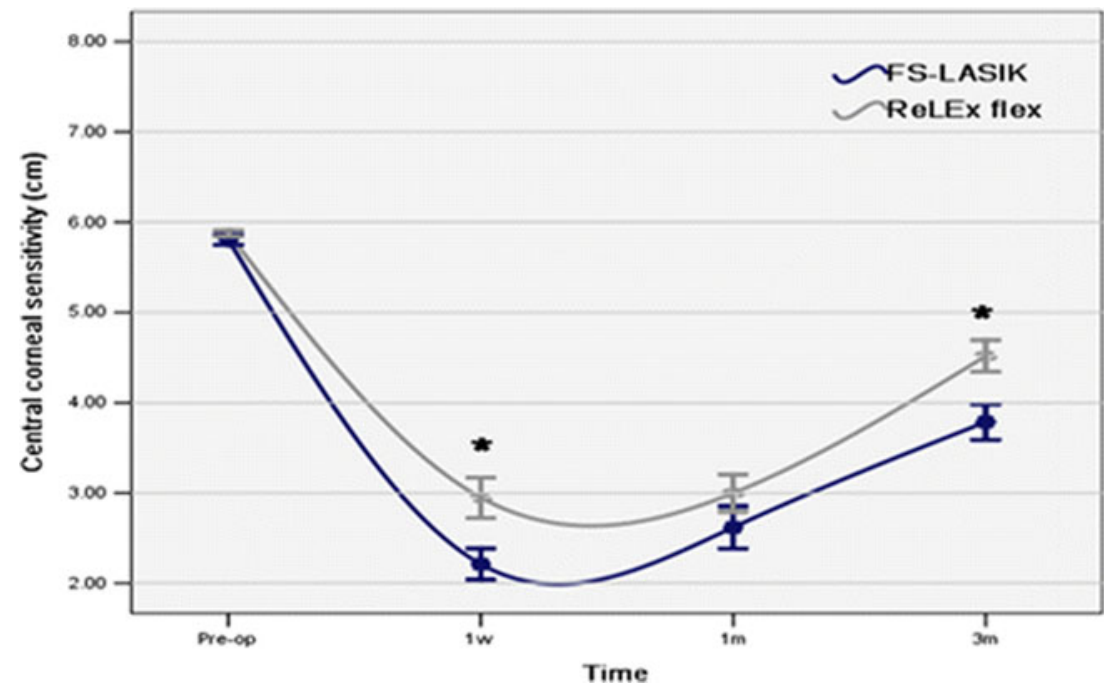

Fig. 2 Comparison in central corneal sensitivity between the FS-LASIK and ReLEx flex groups at 1 week and 1 and 3 months. There were statistical differences between the FS-LASIK group and ReLEx flex group at 1 week and 3 months
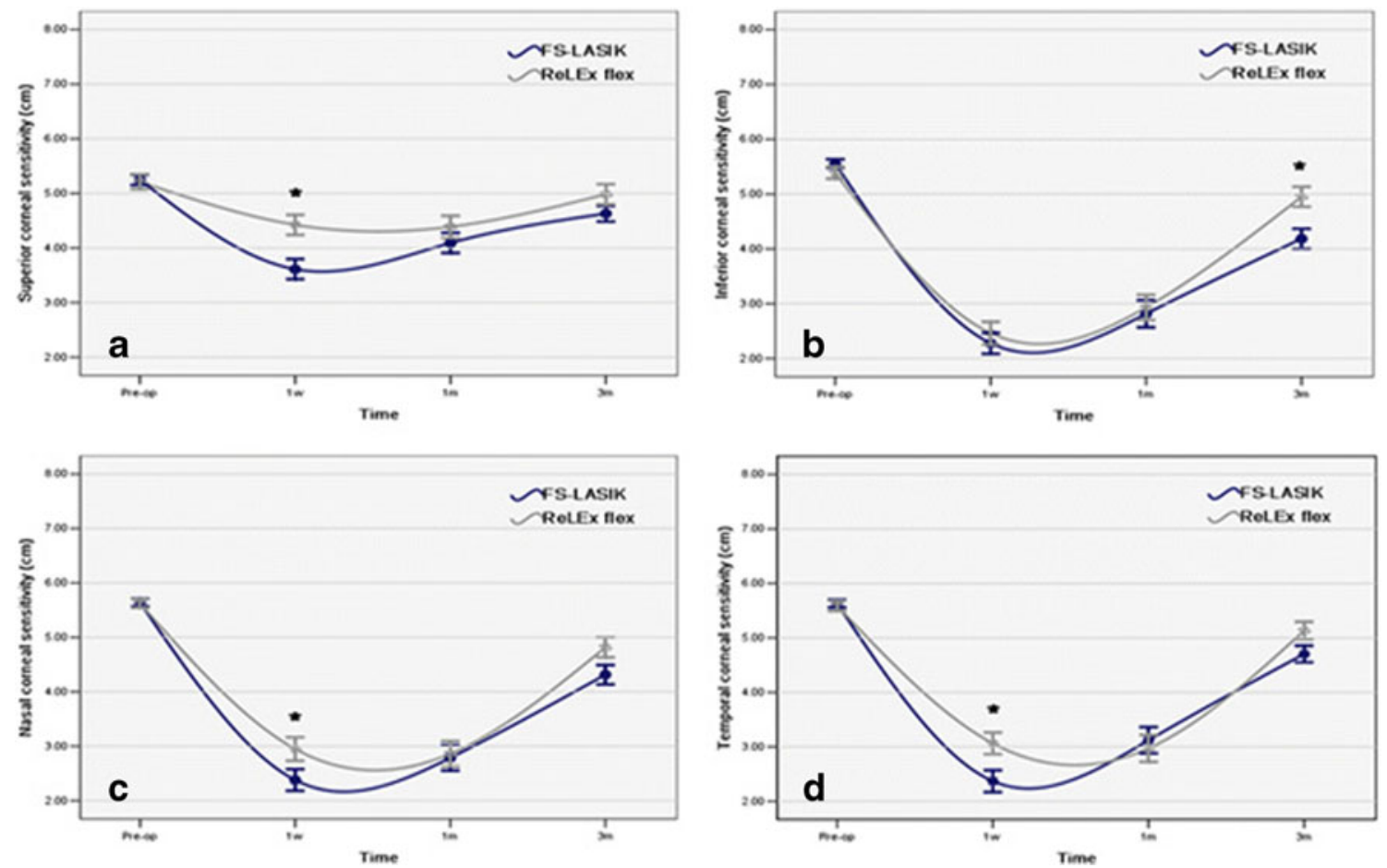

Fig. 3 A comparison of four quadrants - superior (a), inferior (b), nasal (c), and temporal (d) - between the FS-LASIK and ReLEx flex groups at 1 week and 1 and 3 months. There were statistical differences in superior, nasal and temporal corneal sensitivity at 1 week and in inferior sensitivity at 3 months $(\mathrm{P}<0.05$, Mann-Whitney U test $)$ 


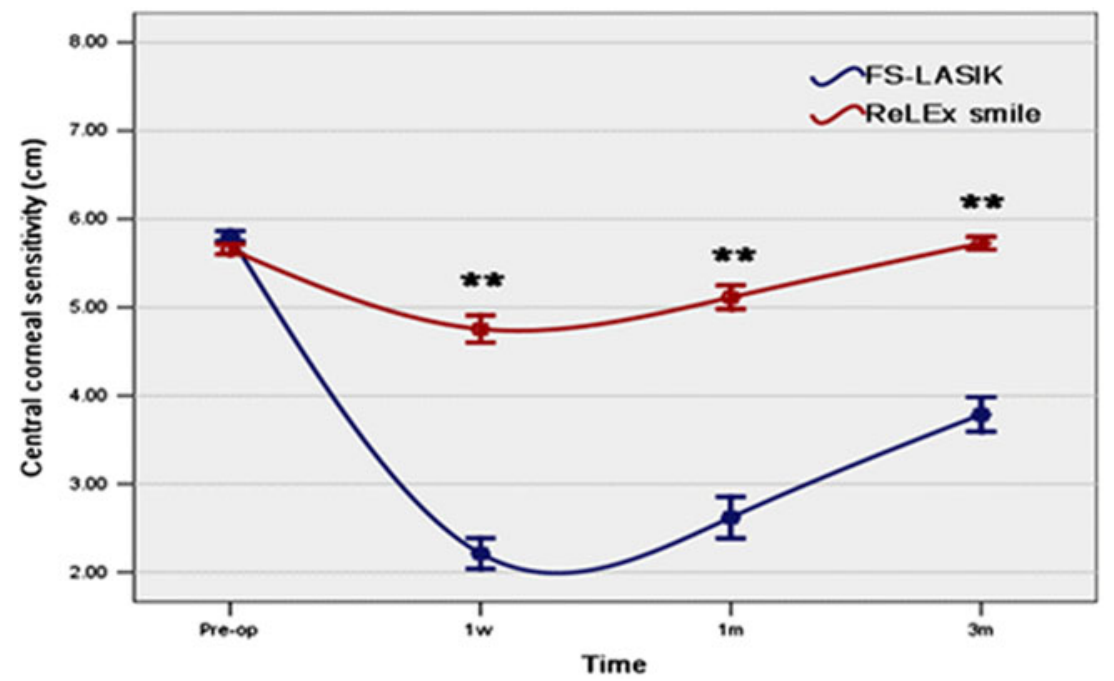

Fig. 4 Comparison of central corneal sensitivity between the FS-LASIK and ReLEx smile groups at 1 week and 1 and 3 months. The corneal sensitivity in the ReLEx smile group was significantly higher than the FS-LASIK group at 1 week, 1 month, and 3 months
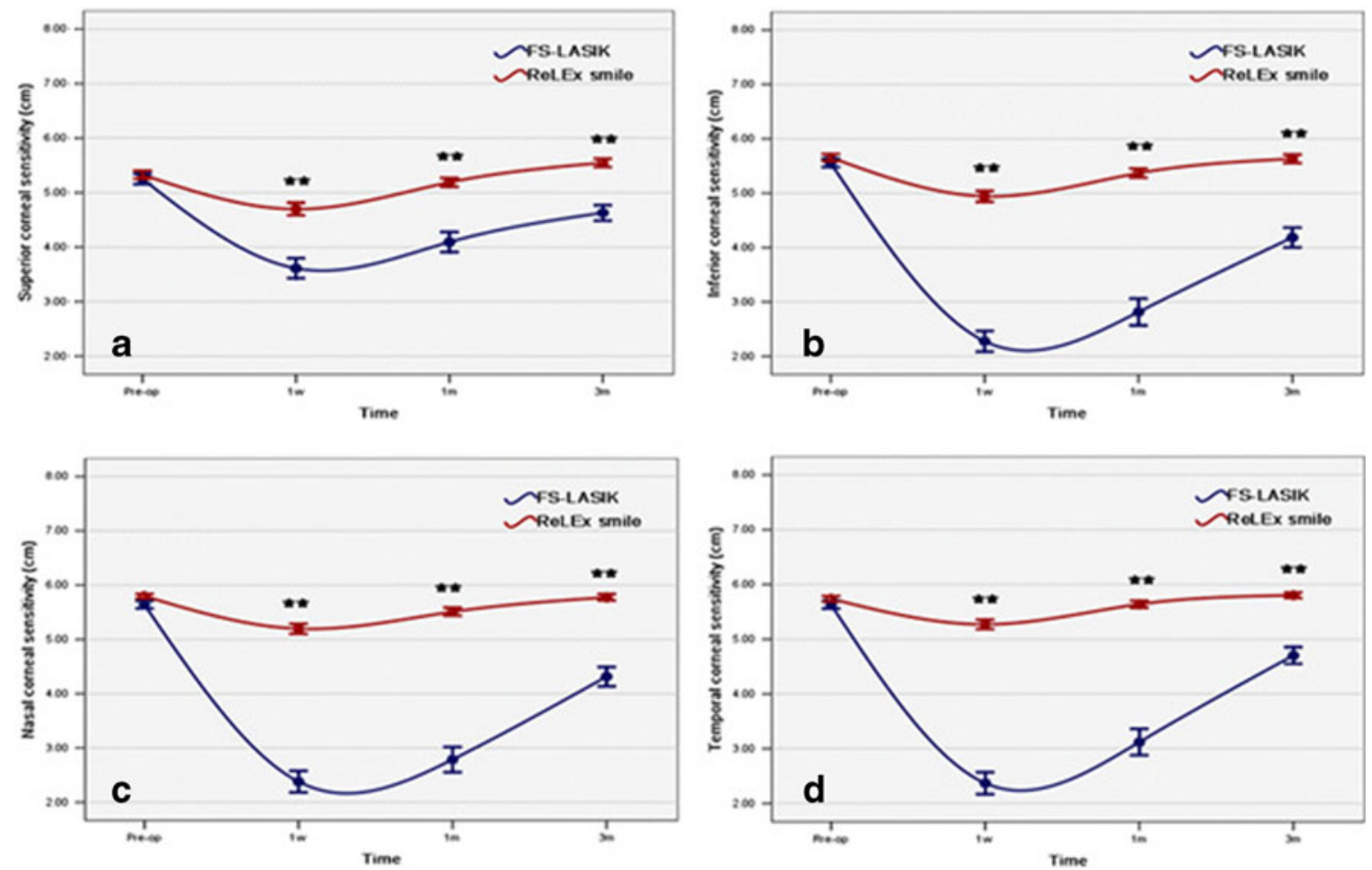

Fig. 5 A comparison of the superior (a), inferior (b), nasal (c), and temporal (d) quadrants in the FS-LASIK group and ReLEx smile groups. There were significantly statistical differences in every quadrant at 1 week, 1 month, and 3 months compared with FS-LASIK surgery 